Bull. Austral. Math. Soc.

VoL. 48 (1993) [101-108]

\title{
A CHARACTERISATION OF RIEMANNIAN FOLIATIONS AND TOTALLY UMBILICAL SUBMANIFOLDS
}

\author{
Ph. Tondeur and L. Vanhecke
}

\begin{abstract}
We discuss characterisations of Riemannian foliations, totally geodesic submanifolds, and totally umbilical submanifolds by sharp inequalities. These derive from the same linear algebraic set up, characterising a linear endomorphism which is a multiple of the identity.
\end{abstract}

\section{General SeT UP}

In the first section we derive two general theorems which will lead to the applications mentioned in the abstract.

Let $V$ be a finite-dimensional real vector space and $g$ an inner product on it. Further, let $V=V_{1} \oplus V_{2}$ be an orthogonal decomposition of $V$ with $\operatorname{dim} V_{1}=m$ and $\operatorname{dim} V_{2}=n$. The induced product on $V_{1}$ will be denoted by $g_{1}$ and that on $V_{2}$ by $g_{2}$.

Next, let $\gamma$ be a symmetric bilinear form on $V_{1}$ with values in $V_{2}$. Then

$$
f: u \mapsto g_{2}(\gamma(u, u), \gamma(u, u))
$$

where $u$ is a unit vector of $V_{1}$, is a function on the unit sphere $S^{m-1}(1)$ in $V_{1}$. Here we identify $V_{1}$ with $\mathbb{R}^{m}$ via an orthonormal basis $\left\{e_{a}, a=1, \ldots, m\right\}$. Further, we put

$$
x=r u=\sum_{a=1}^{m} x_{a} e_{a}
$$

Then we define $F: V_{1} \rightarrow \mathbb{R} ; x \mapsto F(x)$ by

$$
F(x)=r^{4} f(u)=\sum_{a, b, c, d=1}^{m} x_{a} x_{b} x_{c} x_{d} g_{2}\left(\gamma_{a b}, \gamma_{c d}\right)
$$

where $\gamma_{a b}=\gamma\left(e_{a}, e_{b}\right)$.

Received 19th August, 1992

This work was supported by NATO grant CRG.910034.

Copyright Clearance Centre, Inc. Serial-fee code: 0004-9729/93 \$A2.00+0.00. 
Our aim is to compute $\widehat{\Delta} f$ on $S^{m-1}(1)$ where $\widehat{\Delta}$ denotes the Laplace operator on this unit sphere. Therefore, let $\Delta$ denote the Laplace operator on $\mathbb{R}^{m}$. We shall first compute $\Delta F$ and then use the well-known relation (see for example [1])

$$
\Delta=\frac{\partial^{2}}{\partial r^{2}}+\frac{m-1}{r} \frac{\partial}{\partial r}+\widehat{\Delta}_{r}
$$

where $\widehat{\Delta}_{r}$ denotes the Laplacian on $S^{m-1}(r)$. Note that

$$
\Delta F=\sum_{a=1}^{m} \frac{\partial^{2} F}{\partial x_{a}^{2}}
$$

It follows easily that

$$
\begin{aligned}
& \frac{\partial F}{\partial x_{a}}=4 \sum_{b, c, d} x_{b} x_{c} x_{d} g_{2}\left(\gamma_{a b}, \gamma_{c d}\right) \\
& \frac{\partial^{2} F}{\partial x_{a}^{2}}=4 \sum_{c, d} x_{c} x_{d}\left\{g_{2}\left(\gamma_{a a}, \gamma_{c d}\right)+2 g_{2}\left(\gamma_{a c}, \gamma_{a d}\right)\right\}
\end{aligned}
$$

Hence, we obtain

$$
(\Delta F)(x)=4 r^{2} \sum_{a=1}^{m}\left\{g_{2}\left(\gamma\left(e_{a}, e_{a}\right), \gamma(u, u)\right)+2 g_{2}\left(\gamma\left(e_{a}, u\right), \gamma\left(e_{a}, u\right)\right)\right\}
$$

Next, put

$$
\gamma(u, v)=\sum_{i=1}^{n} g_{1}\left(\Gamma_{i} u, v\right) e_{i}
$$

for $u, v \in V_{1}$, in terms of an orthonormal basis $\left\{e_{i}, i=1, \ldots, n\right\}$ of $V_{2}$ and the symmetric endomorphisms $\Gamma_{i}: V_{1} \rightarrow V_{1}, i=1, \ldots, n$. We also define a symmetric non-negative operator $\Gamma^{2}: V_{1} \rightarrow V_{1}$ by

$$
\Gamma^{2} u=\sum_{i=1}^{n} \Gamma_{i}^{2} u
$$

for $u \in V_{1}$. Then (3) can be written in the form

where

$$
\begin{gathered}
(\Delta F)(x)=4 r^{2} \sum_{a=1}^{m}\left\{g_{2}\left(H_{\gamma}, \gamma(u, u)\right)+2 g_{1}\left(\Gamma^{2} u, u\right)\right\} \\
H_{\gamma}=\sum_{a=1}^{m} \gamma\left(e_{a}, e_{a}\right) .
\end{gathered}
$$

Next, using (1) and (2) we get, by putting $r=1$,

$$
(\widehat{\Delta} F)(u)=4\left\{g_{2}\left(H_{\gamma}, \gamma(u, u)\right)+2 g_{1}\left(\Gamma^{2} u, u\right)-(m+2) g_{2}(\gamma(u, u), \gamma(u, u))\right\}
$$


and finally, integration over $S^{m-1}(1)$ yields

$$
\int_{S^{m-1}(1)}\left\{g_{2}\left(H_{\gamma}, \gamma(u, u)\right)+2 g_{1}\left(\Gamma^{2} u, u\right)-(m+2) g_{2}(\gamma(u, u), \gamma(u, u))\right\} d \mu=0
$$

where $d \mu$ is the canonical measure on $S^{m-1}(1)$.

The first general result follows now at once from (6).

THEOREM 1. $\gamma=0$, that is, $V_{1}$ is totally geodesic in $V$, if and only if

$$
g_{2}\left(H_{\gamma}, \gamma(u, u)\right) \geqslant(m+2) g_{2}(\gamma(u, u), \gamma(u, u))
$$

on $S^{m-1}(1)$.

To obtain the second result, we rewrite (6) in the following form :

$$
\begin{gathered}
\int_{S^{m-1}(1)}\left\{g_{2}\left(H_{\gamma}, \gamma(u, u)\right)-g_{1}\left(\Gamma^{2} u, u\right)-(m-1) g_{2}(\gamma(u, u), \gamma(u, u))\right\} d \mu \\
+3 \int_{S^{m-1}(1)}\left\{g_{1}\left(\Gamma^{2} u, u\right)-g_{2}(\gamma(u, u), \gamma(u, u))\right\} d \mu=0 .
\end{gathered}
$$

Since

$$
g_{2}(\gamma(u, u), \gamma(u, u))=\sum_{i=1}^{n} g_{1}\left(\Gamma_{i} u, u\right)^{2},
$$

the Schwarz inequality implies

$$
g_{1}\left(\Gamma^{2} u, u\right) \geqslant g_{2}(\gamma(u, u), \gamma(u, u))
$$

Then we get

THEOREM 2. $V_{1}$ is totally umbilical in $V$, that is, $\Gamma_{i}=\lambda_{i} I$ for $i=1, \ldots, n$, if and only if

$$
g_{2}\left(H_{\gamma}, \gamma(u, u)\right)-g_{1}\left(\Gamma^{2} u, u\right) \geqslant(m-1) g_{2}(\gamma(u, u), \gamma(u, u))
$$

on $S^{m-1}(1)$.

Proof: First, suppose (10) is satisfied. Then we have by (8) and (9)

$$
\sum_{i=1}^{n}\left\{g_{1}\left(\Gamma_{i} u, \Gamma_{i} u\right)-g_{1}\left(\Gamma_{i} u, u\right)^{2}\right\}=0
$$

and hence, by the Schwarz inequality,

$$
g_{1}\left(\Gamma_{i} u, \Gamma_{i} u\right)=g_{1}\left(\Gamma_{i} u, u\right)^{2}
$$

for $i=1, \ldots, n$. Thus, $\Gamma_{i} u$ must be proportional to $u$ for all $u$, which proves the result.

Conversely, let $\Gamma_{i}=\lambda_{i} I, i=1, \ldots, n$. Then we have

$$
\gamma(u, u)=\sum_{i=1}^{n} \lambda_{i} e_{i}, \quad H_{\gamma}=m \sum_{i=1}^{n} \lambda_{i} e_{i}, \quad \Gamma^{2}=\left(\sum_{i=1}^{n} \lambda_{i}^{2}\right) I
$$

and the equality holds in (10). 
REMARK. (6) may also be checked directly by using the integration formulas (see for example $[3,6])$. Then we have

$$
\begin{aligned}
\int_{S^{m-1}(1)} g_{2}\left(H_{\gamma}, \gamma(u, u)\right) d \mu & =\frac{c_{m-1}}{m} g_{2}\left(H_{\gamma}, H_{\gamma}\right)=\frac{c_{m-1}}{m}\left|H_{\gamma}\right|^{2}, \\
\int_{S^{m-1}(1)} g_{1}\left(\Gamma^{2} u, u\right) d \mu & =\frac{c_{m-1}}{m} \text { trace } \Gamma^{2}, \\
\int_{S^{m-1}(1)} g_{2}(\gamma(u, u), \gamma(u, u)) d \mu & =\frac{c_{m-1}}{m(m+2)}\left\{|H|^{2}+2 \text { trace } \Gamma^{2}\right\}
\end{aligned}
$$

where $c_{m-1}$ denotes the volume of $S^{m-1}(1)$.

In the rest of this note we shall consider applications of these two theorems by specialising the symmetric bilinear form $\gamma$. The first one treats foliations and the second one submanifolds of Riemannian manifolds.

\section{ApPLiCATIONS TO FOLIATIONS}

Let $\mathfrak{F}$ be a foliation on a Riemannian manifold $(M, g)$. We refer to $[8,9]$ for more detailed information. We denote vector fields tangent to $\mathfrak{F}$ by $U, V, \ldots$ and vector fields orthogonal (transversal) to $\mathfrak{F}$ by $X, Y, \ldots$ The metric restricted to tangent vectors is denoted by $g_{L}$, and the metric restricted to normal vectors is denoted by $g_{Q}$. For the Lie derivative $\theta(U) g_{Q}$ we have then the following formula $[9,(6.9)]$ :

LEмMA 3. $\left(\theta(U) g_{Q}\right)(X, Y)=-2 g_{L}\left(\alpha^{\perp}(X, Y), U\right)$, where $\alpha^{\perp}$ is the second fundamental form of the normal bundle $L^{\perp}$ of $\mathfrak{F}$.

In terms of the Levi Civita connection $\nabla^{M}$ of $g$ and the tangential projection $\pi^{L}: T M \rightarrow L$, the symmetric bilinear form $\alpha^{L}$ is given by

$$
\alpha^{L}(X, Y)=\frac{1}{2} \pi^{L}\left(\nabla_{X}^{M} Y+\nabla_{Y}^{M} X\right)
$$

$[8,9,(6.8)]$.

Using $\alpha^{\perp}$ for $\gamma$ in Theorem 1 we get the following characterisations of Riemannian foliations, given by $\alpha^{\perp}=0$ according to $[9$, Corollary 6.10].

THEOREM 4. Let $\mathfrak{F}$ be a foliation of codimension $q$ on a Riemannian manifold $(M, g)$. Then $\mathfrak{F}$ is Riemannian (that is, $g$ is bundle-like) if and only if

$$
g_{L}\left(\tau^{\perp}, \alpha^{\perp}(x, x)\right) \geqslant(q+2) g_{L}\left(\alpha^{\perp}(x, x), \alpha^{\perp}(x, x)\right)
$$

for all unit normal vectors $x$ of $\mathfrak{F}$.

Note that $\tau^{\perp}$ is the mean curvature vector field of the normal bundle given by

$$
\tau^{\perp}=\sum_{a=1}^{q} \alpha^{\perp}\left(e_{a}, e_{a}\right)
$$


for an orthonormal frame $\left\{e_{a}, a=1, \ldots, q\right\}$ of transversal vectors $[9,(6.27)]$.

Next, we consider the class of foliations with a holonomy invariant transversal form $\boldsymbol{\nu}$. According to $[\mathbf{9},(6.32)]$, these are characterised by $\tau^{\perp}=0$. Assuming this condition, we rewrite $(6)$ in the form

$$
\begin{gathered}
\int_{S^{q-1}(1)}\left\{(2-\beta) g_{Q}\left(\left(A^{\perp}\right)^{2} x, x\right)-(q+2-\beta) g_{L}\left(\alpha^{\perp}(x, x), \alpha^{\perp}(x, x)\right)\right\} d \mu \\
+\beta \int_{S^{q-1}(1)}\left\{g_{Q}\left(\left(A^{\perp}\right)^{2} x, x\right)-g_{L}\left(\alpha^{\perp}(x, x), \alpha^{\perp}(x, x)\right)\right\} d \mu=0
\end{gathered}
$$

at each point of $M$ and for each unit normal vector $x$ to $\mathfrak{F}$ at this point. Here $\left(A^{\perp}\right)^{2}: L^{\perp} \rightarrow L^{\perp}$ is the endomorphism $\Gamma^{2}$ corresponding to the bilinear form $\alpha^{\perp}$. Then Theorem 2 yields the following result.

Theorem 5. Let $\mathfrak{F}$ be a foliation with holonomy invariant transversal volume on the Riemannian manifold $(M, g)$. Then $\mathfrak{F}$ is Riemannian if and only if there exists a number $\beta$ such that

$$
(2-\beta) g_{Q}\left(\left(A^{\perp}\right)^{2} x, x\right) \geqslant(q+2-\beta) g_{L}\left(\alpha^{\perp}(x, x), \alpha^{\perp}(x, x)\right) \quad \text { for } \beta>0,
$$

or

$$
(2-\beta) g_{Q}\left(\left(A^{\perp}\right)^{2} x, x\right) \leqslant(q+2-\beta) g_{L}\left(\alpha^{\perp}(x, x), \alpha^{\perp}(x, x)\right) \quad \text { for } \beta<0
$$

for each unit normal vector $x$ to $\mathfrak{F}$.

\section{APPLications TO SUBMANIFOLdS}

For a second series of applications we consider submanifolds of a Riemannian manifold. For definitions and more detailed information we refer to [2], for example.

Let $(M, g)$ be a Riemannian manifold of class $C^{\infty}$ and suppose $\operatorname{dim} M=m$. Further, let $\widetilde{\nabla}$ denote its Levi Civita connection and $\widetilde{R}$ its Riemann curvature tensor defined by

$$
\tilde{R}_{X Y}=\tilde{\nabla}_{[X, Y]}-\left[\tilde{\nabla}_{X}, \tilde{\nabla}_{Y}\right]
$$

for tangent vector fields $X, Y$. Next, let $(N, g)$ denote an $n$-dimensional submanifold of $M$ where $g$ also denotes the induced metric. Its Riemann curvature tensor will be denoted by $R$ and the associated Ricci tensor by $\rho$.

Now, let $p \in N$ and let $\left\{e_{A}, A=1, \ldots, m\right\}$ be an orthonormal basis of $T_{p} M$ such that $e_{i}, i=1, \ldots, n$ are tangent to $N$ and $e_{n+1}, \ldots, e_{m}$ normal to $N$ at $p$. Further, let $\sigma$ denote the second fundamental form of $N$ and put, at $p$,

$$
g\left(\sigma(x, y), e_{a}\right)=-g\left(S_{a} x, y\right), \quad a=n+1, \ldots, m
$$


for $x, y \in T_{p} N$. Here $S_{a}$ denotes the shape operator corresponding to $e_{a}$. For $u \in T_{p} N$, the Gauss equation yields

$$
\begin{aligned}
\rho(u, u) & =\sum_{i=1}^{n} \widetilde{R}_{u e_{i} u e_{i}}+g(H, \sigma(u, u))-\sum_{a=n+1}^{m} g\left(S_{a} u, S_{a} u\right) \\
& =\widetilde{\rho}(u, u)-\sum_{a=n+1}^{m} \widetilde{R}_{u e_{a} u e_{a}}+g(H, \sigma(u, u))-\sum_{a=n+1}^{m} g\left(S_{a} u, S_{a} u\right)
\end{aligned}
$$

where $\tilde{\rho}$ is the Ricci tensor of $(M, g)$ and $H$ the mean curvature vector field of $N$ in $M$.

For an arbitrary unit vector $u \in T_{p} N,(6)$ becomes, using $\gamma=\sigma$ and (11),

$$
\int_{S^{n-1}(1)}\left\{\rho(u, u)-\sum_{i=1}^{n} \tilde{R}_{u e_{i} u e_{i}}-(n+2) g(\sigma(u, u), \sigma(u, u))\right\} d \mu+3 \int_{S^{n-1}(1)} g\left(S^{2} u, u\right) d \mu=0
$$

and (8) may be written as

$$
\begin{gathered}
\int_{S^{n-1}(1)}\left\{\rho(u, u)-\sum_{i=1}^{n} \tilde{R}_{u e_{i} u e_{i}}-(n-1) g(\sigma(u, u), \sigma(u, u))\right\} d \mu \\
+3 \int_{S^{n-1}(1)}\left\{g\left(S^{2} u, u\right)-g(\sigma(u, u), \sigma(u, u))\right\} d \mu=0
\end{gathered}
$$

where $S^{2}$ corresponds to $\Gamma^{2}$. The relations (12), (13) yield at once

THEOREM $6 . N$ is a totally geodesic submanifold if and only if

$$
\rho(u, u) \geqslant(n+2) g(\sigma(u, u), \sigma(u, u))+\sum_{i=1}^{n} \widetilde{R}_{u e_{i} u e_{i}}
$$

for all unit tangent vectors $u$.

TheOrem 7. $N$ is a totally umbilical submanifold if and only if

$$
\rho(u, u) \geqslant(n-1) g(\sigma(u, u), \sigma(u, u))+\sum_{i=1}^{n} \widetilde{R}_{u e_{i} u e_{i}}
$$

for all unit tangent vectors $u$.

As a special case we then get

Corollary 8. Let $(M, g)$ be a space of constant curvature $c$. Then $N$ is a totally geodesic submanifold if and only if

$$
\rho(u, u) \geqslant(n-1) c+(n+2) g(\sigma(u, u), \sigma(u, u))
$$


for all unit tangent vectors $u$.

Corollary 9. Let $(M, g)$ be a space of constant curvature $c$. Then $N$ is a totally umbilical submanifold if and only if

$$
\rho(u, u) \geqslant(n-1)\{c+g(\sigma(u, u), \sigma(u, u))\}
$$

for all unit tangent vectors $u$.

REMARK. For $c=0$ and $n=m-1$ we obtain the result provided in [4] and used to obtain a characterisation for a compact and connected hypersurface in $\mathbb{R}^{n+1}$ to be a sphere in terms of lower bounds on the Ricci curvature.

Finally, we consider some special cases in almost Hermitian theory. For more details about submanifolds in this context we refer to [7]. Let $(M, g, J)$ be a Kähler manifold and let $N$ be a holomorphic submanifold of $M$. Then $N$ is minimal and we get

THEOREM 10. Let $N$ be a holomorphic submanifold of a Kähler manifold $(M, g, J)$. Then $N$ is totally geodesic if and only if

$$
\rho(u, u) \geqslant(n-1) g(\sigma(u, u), \sigma(u, u))+\sum_{i=1}^{n} \widetilde{R}_{u e_{i} u e_{i}}
$$

for all unit tangent vectors $u$.

Note that the same result holds when $(M, g, J)$ is a quasi-Kähler manifold, that is, an almost Hermitian manifold satisfying

$$
\left(\nabla_{X} J\right) Y+\left(\nabla_{J X} J\right) J Y=0
$$

for all tangent vectors $X, Y[5]$.

Next, let $(M, g, J)$ be a complex space form, that is, a Kähler manifold of constant holomorphic sectional curvature, say $c$. Further, we note that the second fundamental form $\sigma$ of a holomorphic submanifold $N$ in a Kähler manifold satisfies

$$
\sigma(J u, u)=J \sigma(u, u), \quad \sigma(J u, J u)+\sigma(u, u)=0
$$

for any tangent vector $u$. This and the Gauss equation yield that the holomorphic sectional curvature $H(u)$ of $N$ corresponding to the unit vector $u$ is given by

So since

$$
\begin{gathered}
H(u)=c-2 g(\sigma(u, u), \sigma(u, u)) . \\
\sum_{i=1}^{n} \widetilde{R}_{u e_{i} u e_{i}}=\frac{n+2}{4} c
\end{gathered}
$$

in the complex space form $M$, we obtain 
Corollary 11. Let $N$ be a holomorphic submanifold of a Kähler manifold of constant holomorphic sectional curvature $c$. Then $N$ is totally geodesic if and only if for any unit vector $u$ tangent to $N$ we have

$$
\rho(u, u) \geqslant \frac{n+2}{4} c+(n-1) g(\sigma(u, u), \sigma(u, u))
$$

or equivalently,

$$
\rho(u, u) \geqslant \frac{3 n}{4} c-\frac{n-1}{2} H(u)
$$

\section{REFERENCES}

[1] M. Berger, P. Gauduchon and E. Mazet, Le spectre d'une variété riemannienne, Lecture Notes in Mathematics 194 (Springer-Verlag, Berlin, Heidelberg, New York, 1971).

[2] B.Y. Chen, Geometry of submanifolds, Pure Appl. Math. 22 (Marcel Dekker, New York, 1973).

[3] B.Y. Chen and L. Vanhecke, 'Differential geometry of geodesic spheres', J. Reine Angew. Math. 325 (1981), 28-67.

[4] S. Deshmukh, 'A note on hypersurfaces in a Euclidean space', Geom. Dedicata 34 (1990), 101-103.

[5] A. Gray, 'Minimal varieties and almost Hermitian submanifolds', Michigan Math. J. 12 (1965), 273-287.

[6] A. Gray and L. Vanhecke, 'Riemannian geometry as determined by the volumes of small geodesic balls', Acta Math. 142 (1979), 157-198.

[7] K. Ogiue, 'Differential geometry of Kaehler submanifolds', Adv. in Math. 13 (1974), 73-114.

[8] B.L. Reinhart, Differential geometry of foliations, Ergebnisse der Mathematik 99 (SpringerVerlag, Berlin, Heidelberg, New York, 1983).

[9] Ph. Tondeur, Foliations on Riemannian manifolds, Universitext (Springer-Verlag, Berlin, Heidelberg, New York, 1988).

Department of Mathematics

University of Illinois at Urbana-Champaign

Urbana II. 61801

United States of America
Department of Mathematics

Katholieke Universiteit Leuven

Celestijnenlaan 200B

B-3001 Leuven

Belgium 